\title{
AN UPPER BOUND FOR THE PERMANENT OF A FULLY INDECOMPOSABLE MATRIX
}

\author{
THOMAS H. FOREGGER
}

ABSTRACT. Let $A$ be an $n \times n$ fully indecomposable matrix with nonnegative integer entries and let $\sigma(A)$ denote the sum of the entries of $A$. We prove that $\operatorname{per}(A) \leq 2^{\sigma(A)-2 n}+1$ and give necessary and sufficient conditions for equality to hold.

1. Introduction. The permanent of an $n \times n$ matrix $A=\left(a_{i j}\right)$ is defined by

$$
\operatorname{per}(A)=\sum_{\sigma \in S_{n}} \prod_{i=1}^{n} a_{i \sigma(i)}
$$

where the summation is over all elements of the symmetric group. If $A=\left(a_{i j}\right)$ is an $n \times n(0,1)$ matrix, then $0 \leq \operatorname{per}(A) \leq \Pi_{i=1}^{n} r_{i}$ where $r_{i}=\sum_{j=1}^{n} a_{i j}, i=$ $1, \cdots, n$. Improvements of this upper bound have been made by several authors; see [1]-[4]. The first was in 1963 by Minc, who showed that $\operatorname{per}(A) \leq$ $\Pi_{i=1}^{n}\left(r_{i}+1\right) / 2$, with equality if and only if $A$ is a permutation matrix. Here we give an easily computed upper bound for $\operatorname{per}(A)$ in terms of $N$, the number of positive entries in $A$, and $n$, the dimension of $A$.

A conjecture of E. J. Roberts [5, p. 78] states that if $A$ is an $n \times n$ nearly decomposable $(0,1)$ matrix with $N$ positive entries then $\operatorname{per}(A) \leq$ $2^{N-2 n}+1$. We prove a stronger result and determine for which matrices $A$ equality holds.

\section{Results.}

Theorem 1. Let $A$ be an $n \times n(0,1)$ matrix with all row sums $r_{i} \geq 3$. Let $N$ be the number of positive entries in $A$. Then $\operatorname{per}(A)<2^{N-2 n}$.

Proof. It can be verified by induction that for $k \geq 3,(k+1) / 2 \leq 2^{k-2}$. Since $r_{i} \geq 3$ for all $i, A$ is not a permutation matrix. Hence by Minc's result

Received by the editors August 25, 1973 and, in revised form, April 8, 1974. AMS (MOS) subject classifications (1970). Primary 15A15; Secondary 15A36, $15 A 45,15 A 48$.

Key words and phrases. Permanent, fully indecomposable, $(0,1)$ matrix. 


$$
\operatorname{per}(A)<\prod_{i=1}^{n} \frac{r_{i}+1}{2} \leq \prod_{i=1}^{n} 2^{r_{i}-2}=2^{\Sigma_{i=1}^{n} r_{i}-2 n}=2^{N-2 n} .
$$

For $A$ an $n \times n$ matrix, let $\sigma(A)$ denote the sum of all entries of $A$. A nonnegative $n$-square matrix $A$ is said to be fully indecomposable if $A$ does not contain an $s \times(n-s)$ zero matrix, $1 \leq s \leq n-1$. As a consequence of the Frobenius-König theorem, every $(n-1)$-square submatrix of such a matrix must have a positive diagonal. Hence, if $A(i \mid j)$ denotes the submatrix obtained from $A$ by deleting the $i$ th row and $j$ th column, we must have $\operatorname{per} A(i \mid j)>0$.

Lemma 1. Let $A$ be an $n \times n$ fully indecomposable matrix with nonnegative integer entries. Suppose for some $i, j a_{i j} \geq 2$. Then

$$
\operatorname{per}(A) \leq 2 \operatorname{per}\left(A-E_{i j}\right)-1
$$

$\left(E_{i j}\right.$ denotes the $n \times n$ matrix whose $(i, j)$ entry is 1 and whose other entries are 0).

Proof. Since $A$ is fully indecomposable there is an $l \neq j$ such that $a_{i l} \geq 1$. Expanding the permanent by its $i$ th row, we have

$$
\begin{aligned}
\operatorname{per}(A) & =a_{i j} \operatorname{per}(A(i \mid j))+a_{i l} \operatorname{per}(A(i \mid l))+\sum_{k \neq i, l} a_{i k} \operatorname{per}(A(i \mid k)) \\
& \geq 2 \operatorname{per}(A(i \mid j))+1
\end{aligned}
$$

or $\operatorname{per}(A(i \mid j)) \leq(\operatorname{per}(A)-1) / 2$. Expanding $\operatorname{per}(A)$ by its $i$ th row again, we have

$$
\operatorname{per}(A)=\operatorname{per}\left(A-E_{i j}\right)+\operatorname{per}(A(i \mid j)) \leq \operatorname{per}\left(A-E_{i j}\right)+(\operatorname{per}(A)-1) / 2
$$

from which (1) follows.

The proof shows that equality holds in (1) if and only if $a_{i j}=2, a_{i l}=1$, $\operatorname{per}(A(i \mid l))=1$, and $a_{i k}=0$ for $k \neq i, l$. Moreover by expanding the permanent of $A$ by the $j$ th column we see that there exists $m \neq i$ such that $a_{m j}=1$ and $a_{k j}=0$ for $k \neq m, i$.

Theorem 2. Let $n \geq 1$ and suppose $A$ is an $n \times n$ fully indecomposable matrix with nonnegative integer entries. Then

$$
\operatorname{per}(A) \leq 2^{\sigma(A)-2 n}+1
$$

For $n>1$ equality holds if and only if there exist permutation matrices $P$ and $Q$ such that 


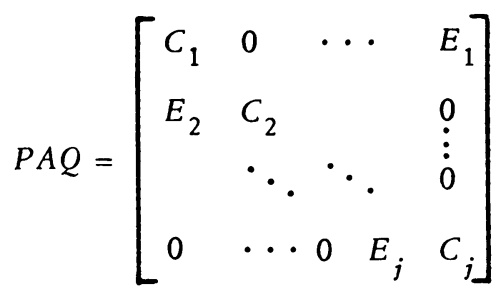

where $C_{i}$ is $n_{i}$-square, $E_{i}$ is $n_{i} \times n_{i-1}(i=2, \cdots, j)$ and $E_{1}$ is $n_{1} \times n_{j}$. Each $E_{i}$ is a $(0,1)$ matrix containing exactly one 1 . Each $C_{i}$ is the sum of an identity matrix and a full cycle permutation matrix or is $\left(x_{11}\right)$ where $x_{11}$ equals 1 or 2 . For $n=1$ equality holds for the matrix (a) where $a=2$ or $a=3$.

Proof. We proceed by induction on $n$. Let $A$ be an $n \times n$ fully indecomposable matrix with nonnegative integer entries. If $n=1$ the result is easily verified, so we assume $n \geq 2$. If there exists $a_{i j} \geq 2$ set $A_{1}=A-E_{i j}$. Then $\sigma\left(A_{1}\right)=\sigma(A)-1$ and by Lemma $1, \operatorname{per}(A) \leq 2 \operatorname{per}\left(A_{1}\right)-1$. If $A_{1}$ has an entry $a_{k l} \geq 2$, set $A_{2}=A_{1}-E_{k l}$. Then $\operatorname{per}\left(A_{1}\right) \leq 2 \operatorname{per}\left(A_{2}\right)-1$ so that $\operatorname{per}(A) \leq$ $2^{2} \operatorname{per}\left(A_{2}\right)-\left(2^{2}-1\right)$.

Repeated application of Lemma 1 gives a sequence $A=A_{0}, A_{1}, A_{2}, \cdots$ of fully indecomposable matrices with nonnegative integer entries such that $\operatorname{per}(A) \leq 2^{j} \operatorname{per}\left(A_{j}\right)-\left(2^{j}-1\right)$ and $\sigma\left(A_{j}\right)=\sigma(A)-j(j=0,1,2, \cdots)$.

Eventually we obtain a $(0,1)$ matrix $B=A_{m}(m \geq 0)$. Let $r_{i}$ be the ith row sum of $B(i=1,2, \cdots, n)$. There are two cases to consider.

(i) $r_{i} \geq 3$ for all $i$. Then by Theorem 1 per $(B) \leq 2^{\sigma(B)-2 n}-1$ so that $\operatorname{per}(A) \leq 2^{m}\left(2^{\sigma(B)-2 n}-1\right)-\left(2^{m}-1\right)=2^{\sigma(A)-2 n}+1-2^{m+1}<2^{\sigma(A)-2 n}+1$.

(ii) There is an $i$ such that $r_{i}=2$, say $i=1$. Let the 1 's in row 1 of $B$ be in columns 1 and 2. Form a new matrix $B^{\prime}$ by adding together columns 1 and 2 of $B$ and deleting row $1 . B^{\prime}$ is a fully indecomposable $(n-1)$-square matrix and $\operatorname{per}\left(B^{\prime}\right)=\operatorname{per}(B), \sigma\left(B^{\prime}\right)=\sigma(B)-2 . B^{\prime}$ has entries in $\{0,1,2\}$ so by the induction hypothesis

$$
\operatorname{per}(B)=\operatorname{per}\left(B^{\prime}\right) \leq 2^{\sigma\left(B^{\prime}\right)-2(n-1)}+1=2^{\sigma(B)-2 n}+1
$$

Hence

$$
\operatorname{per}(A) \leq 2^{m}\left(2^{\sigma(B)-2 n}+1\right)-\left(2^{m}-1\right)=2^{\sigma(A)-2 n}+1
$$

If equality holds in (2), the proof shows that for $n \geq 2$ the following must hold: 
(a) All entries of $A$ are less than or equal to 2 and if $a_{i j}=2$ then the $i$ th row sum and $j$ th column sum of $A$ are both equal to 3 .

(b) $B$ has a row sum equal to 2 .

(c) $\operatorname{per}\left(B^{\prime}\right)=2^{\sigma\left(B^{\prime}\right)-2(n-1)}+1$.

By the induction hypothesis there exist permutation matrices $P^{\prime}$ and $Q^{\prime}$ such that

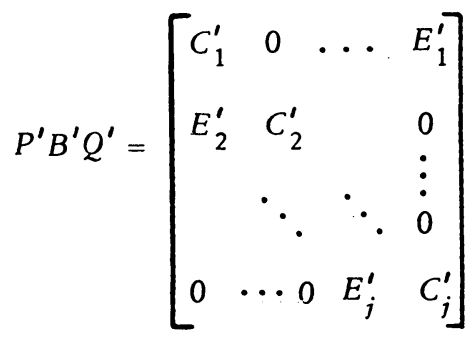

where $C_{i}^{\prime}, E_{i}^{\prime}$ are as described in the statement of the theorem. This implies that no row or column sum of $B^{\prime}$ exceeds 3 , so that each row and column of $B^{\prime}$ has at most three positive entries. Thus $B$ must have only two or three 1 's in columns 1 and 2 among the rows $2,3, \cdots, n$. Hence one of columns 1 and 2 of $B$ has only two 1's. It follows that after permuting rows and columns $B$ must have the form (3). We must now replace certain 1's of $B$ by 2's to obtain $A$.

Suppose first that some $C_{i}$ has a 2 (after replacement) and that $n_{i}>1$. Without loss of generality we can assume $i=1$ and that the 2 is in row $n_{1}$, column $n$. Then $a_{1 n_{1}}=a_{n_{1}, n_{1}-1}=1$. Thus

$A=\left[\begin{array}{ccccc}C_{1} & 0 & \cdots & 0 & E_{1} \\ E_{2} & C_{2} & & & 0 \\ 0 & E_{3} & C_{3} & & \vdots \\ \vdots & & \ddots & \ddots & \\ 0 & \cdots & & E_{l} & C_{l}\end{array}\right]$ where $C_{1}=\left[\begin{array}{ccccc}y_{1} & & & & 1 \\ x_{2} & y_{2} & & & \vdots \\ & \ddots & \ddots & & \\ & & x_{n_{1}-1} & y_{n_{1}-1} & 0 \\ & & & 1 & 2\end{array}\right]$.

Then

$$
1=\operatorname{per} A\left(1 \mid n_{1}\right) \geq \prod_{i=2}^{n_{1}^{-1}} x_{i} \prod_{j=2}^{l} \operatorname{per}\left(C_{j}\right) \geq 1
$$

so that $x_{2}=\cdots=x_{n_{1}-1}=1, C_{j}=(1)(j=2, \cdots, l)$ and $l=n-n_{1}+1$. Let $e_{j}$ be the positive entry of $E_{j}(j=1, \cdots, l)$, let $e_{1}$ be in row $i_{0}$, column $n$, and let $e_{2}$ be in row $n_{1}+1$, column $i$. We show that $i_{0} \leq i$. Suppose $i_{0}>i$. Then 
so that

$$
2^{\sigma(A)-2 n}+1=\operatorname{per}(A)=2 \prod_{j=1}^{n_{1}^{-1}} y_{j}+1+\prod_{j=1}^{l} e_{j}
$$

$$
2^{\Sigma y_{j}+\Sigma e_{j}+2-n} \leq 2^{\Sigma y_{j}-\left(n_{1}-1\right)+1}+2^{\Sigma e_{j}-\left(n-n_{1}+1\right)}
$$

or

$$
1 \leq 2^{n-n_{1}-\Sigma e_{j}}+2^{n_{1}-3-\Sigma y_{j}}
$$

Now $\Sigma e_{j} \geq n-n_{1}+1$ and $\Sigma y_{j} \geq n_{1}-1$ so that $n-n_{1}-\Sigma e_{j} \leq-1$ and $n_{1}-3-\Sigma y_{j} \leq-2$. Hence $1 \leq 2^{-1}+2^{-2}=3 / 4$ which is a contradiction. Thus $i_{0} \leq i$. We again calculate

$$
2^{\sigma(A)-2 n}+1=\operatorname{per}(A)=2 \prod y_{i}+1+2\left(\prod_{e_{j}}\right)\left(\prod_{y_{j}}\right) \frac{1}{Y}
$$

where $Y=\Pi_{j=i_{0}}^{i} y_{j}$. Thus

$$
2^{\Sigma e_{j}+\Sigma y_{j}+1-n} \leq 2^{\Sigma y_{j}-\left(n_{1}-1\right)}+\frac{1}{Y} 2^{\Sigma e_{j}+\Sigma y_{j}-n}
$$

or

$$
1 \leq 2^{n-n_{1}-\Sigma e} j+\frac{1}{Y} 2^{-1} \leq 2^{-1}+2^{-1}=1 \text {. }
$$

Therefore equality must hold throughout. This means that $\Sigma e_{j}=n-n_{1}+1$ so each $e_{j}=1$. Also $Y=1$ so $y_{i_{0}}=\cdots=y_{i}=1$ and $y_{j}=1$ or 2 whenever $j<i_{0}$ or $j>i$. It follows that the submatrix formed by rows $i_{0}, \cdots, i, n_{1}+1$, $\cdots, n$ and colums $i_{0}, \cdots, i, n_{1}+1, \cdots, n$ is the sum of an identity matrix and a full cycle permutation matrix. Thus we may rearrange the rows and columns of $A$ to get $A$ into the form (3), where each $C_{i}=\left(y_{i}^{\prime}\right), y_{i}^{\prime}=1$ or 2 , $i=1, \cdots, j-1$, and at least one $y_{i}^{\prime}$ will be equal to 2 . The matrix $C_{j}$ will be the sum of an identity matrix and a full cycle permutation matrix.

The remaining case is when each $C_{i}$ is the sum of an identity matrix and a full cycle permutation matrix or is $\left(x_{11}\right)$ where $x_{11}$ equals 1 or 2 . Let $e_{i}$ be the positive entry of $E_{i}(i=1,2, \cdots, j)$. If all $e_{i}$ 's are 1 then $A$ has the form (3). Suppose $e_{l}>1$. Then $\sigma(A)>2 n$ and $C_{l}=C_{l-1}=(1)$. We have

$$
2^{\sigma(A)-2 n}+1=\operatorname{per}(A)=\prod_{k=1}^{j} \operatorname{per}\left(C_{k}\right)+2 \prod_{k=1 ; k \neq l, l-1}^{j} e_{k} .
$$

Hence $\Pi_{k=1}^{j}$ per $\left(C_{k}\right)$ is an odd integer. By assumption on the $C_{i}$ 's, it must be that $C_{i}=(1)$ for all $i$. But then $A$ can be rearranged to get $A=$ $\operatorname{diag}\left(e_{1}, \cdots, e_{j}\right)+P$, where $e_{i}=1$ or 2 and $P$ is a full cycle permutation 
matrix. Since $A$ now has the form (3), this completes the proof.

Now suppose $A$ is a $(0,1)$ matrix with row sums $r_{1}, r_{2}, \cdots, r_{n}$. If all the $r_{i}$ 's are greater than 2 then Minc's result gives a better upper bound for the permanent than Theorem 2. For this case the bound obtained from the result of Nijenhuis and Wilf [4] will be better than Minc's. However, if $r_{i}=2$ for some $i$ then Theorem 2 may give a better bound than either of these. For example, the matrix $A$ below has $\operatorname{per}(A)=3$. Minc's bound is $2(3 / 2)^{3}=6.25$, the Nijenhuis and Wilf bound is about 7.29, and Theorem 2 gives $\operatorname{per}(A)=3$.

The matrix $A(\epsilon)$ below (with $\epsilon=0$ ) shows that the assumption of full indecomposability in Theorem 2 cannot be dropped. The same example with $\epsilon>0$ and sufficiently small shows that Theorem 2 does not hold for arbitrary nonnegative matrices.

$$
A=\left[\begin{array}{llll}
1 & 0 & 1 & 0 \\
1 & 1 & 0 & 1 \\
0 & 1 & 1 & 0 \\
0 & 0 & 1 & 1
\end{array}\right], \quad A(\epsilon)=\left[\begin{array}{lll}
1 & 1 & 0 \\
1 & 1 & \epsilon \\
0 & \epsilon & 1
\end{array}\right] .
$$

\section{REFERENCES}

1. Henryk Minc, Upper bounds for permanents of $(0,1)$-matrices, Bull. Amer. Math. Soc. 69 (1963), 789-791. MR $27 \# 5777$.

2. - An inequality for permanents of $(0,1)$-matrices, J. Combinatorial Theory 2 (1967), 321-326. MR 35 \#2765.

3. W. B. Jurkat and H. J. Ryser, Matrix factorizations of determinants and permanents, J. Algebra 3 (1966), 1-27. MR 33 \#4075.

4. A. Nijenhuis and H. S. Wilf, On a conjecture of Ryser and Minc, Nederl. Akad. Wetensch. Proc. Ser. A 73 = Indag. Math. 32 (1970), 151-157. MR 42 \#4566.

5. Edwin J. Roberts, The fully indecomposable matrix and its as sociated bipartite graph-an investigation of combinatorial and structural properties, NASA Technical Memorandum TMX-58037, January 1970.

DEPARTMENT OF MATHEMATICS, UNIVERSITY OF WISCONSIN, MADISON, WISCONSIN 53706

Current address: Bell Telephone Laboratories, Murray Hill, New Jersey 07974 